\title{
La escuela privada en Entre Ríos: análisis de los motivos de las familias en la elección de escuela secundaria
}

\author{
VIRGINIA KUMMER ${ }^{(1)}$
}

Fecha de recepción: 09/03/2020

Fecha de aceptación: 26/06/2020

Resumen. Este artículo analiza los motivos de las familias que envían sus hijos a la escuela secundaria pública de gestión privada. El estudio se focaliza en la ciudad de Paraná, capital de la provincia de Entre Ríos. La relevancia del tema es ciertamente alta, dado que se trata de una problemática que ha atravesado en toda su historia el sistema educativo argentino y continúa teniendo hoy, plena actualidad. La realidad muestra que, al menos, un número importante de provincias argentinas, está caracterizado por el éxodo de los sectores medios hacia la oferta privada, no siendo una particularidad exclusiva de la escuela secundaria, sino también de otros niveles del sistema educativo. Metodológicamente, el análisis se basa en un conjunto de entrevistas semi-estructuradas a padres con hijos en escuelas privadas secundarias. A tal fin, fueron seleccionadas mediante un muestreo intencional, cuatro escuelas privadas de la ciudad de Paraná, consideradas representativas a nivel teórico y definidas por su singularidad y carácter idiosincrático. Los resultados indican que la escuela privada oferta una educación diferenciada, que es considerada por las familias una estrategia adecuada para proyectar el futuro de sus hijos. Motivos como la tradición familiar, la disciplina y contención social, la laicidad y el tamaño de la institución, se encuentran en el origen de esta elección.
Palabras clave. escuela privada $\cdot$ motivos $\cdot$ estrategias familiares $\cdot$ nivel secundario $\cdot$ educación diferenciada

(1) Universidad Nacional de Entre Ríos (UNER). Profesora y Licenciada en Ciencias de la Educación, Orientación en Planeamiento y Administración de la Educación. Magister en Educación con Orientación en Historia y Prospectiva (FCDU/ UNER), Doctora en Ciencias Sociales (UNER). Titular Ordinaria de Metodología de la Investigación Social, Licenciatura en Comunicación Social (FCEDU/UNER) y Metodología de la Investigación, Licenciatura en Diseño de la Comunicación Visual (FADU/UNL). Ha participado en proyectos de investigación vinculados al área educación-política y sociedad. virginia.kummer@uner.edu.ar 


\section{Private School in Entre Ríos: Analyzing Families' Reasons for Choosing Secondary Schools for their Children}

\begin{abstract}
This article analyzes the reasons why families choose to send their children to public secondary schools that are managed by privates. The study focused on the city of Paraná, capital of the province of Entre Ríos, Argentina. This issue has gained relevance for constituting a problem that has been always present throughout the history of Argentine educational system. Reality shows that, at least, an important number of Argentine provinces are characterized by an exodus of middle-class sectors to the private offer, this being in no way an exclusive feature noticed for secondary schools but also for other levels of the education system. As regards the applied methodology, the analysis was based on a set of semi-structured interviews held with
\end{abstract}

parents of children attending private schools. To this end, four private schools in the city that were considered representative at a theoretical level and defined by their uniqueness and idiosyncratic character were selected by means of an intentional sampling. Results indicated that private schools offer differentiated education, which is regarded by families as an appropriate strategy to plan their children's future. Different reasons such as family tradition, discipline, social support, secularism, and the institution size constitute the roots of this choice.

Keywords. private schools $\cdot$ reasons . family strategies $\cdot$ secondary level . differentiated education

\section{Introducción}

El análisis de los motivos de las familias para elegir la escuela de sus hijos, supone analizar la reflexividad de los padres (Van Zanten, 2007) en su carácter de ciudadanos y actores sociales. El conocimiento de las estrategias que están poniendo en juego, es fundamental para el campo de las políticas educativas, las que constituyen sin duda, una oportunidad para que el Estado pueda mediar e intervenir en las dinámicas societales, sobre todo, en aquellas que generan resultados no siempre deseables para la equidad del sistema educativo.

Bourdieu (2011) subraya la importancia de la familia conformada para y por la acumulación y la transmisión, en las estrategias educativas. Advierte que, para comprender las estrategias colectivas de las familias es necesario conocer el volumen y la estructura del capital que ellas tienen para transmitir, y por lo tanto, la posición de cada una en la estructura de distribución 
(2) De ahora en adelante se utilizará la expresión educación privada con el fin de agilizar la lectura del texto. Del mismo modo se utiliza el genérico escuela en el sentido de toda institución que imparta educación. de las diferentes formas de capital. Para el sociólogo francés, capacidad, estrategia y recursos se encuentran íntimamente ligados. En la medida en que se acumulan recursos -económicos, culturales, sociales- se modifica la capacidad de una familia de dar respuesta a los problemas planteados en la educación de sus hijos, lo cual también ocurrirá en la medida en que se observan y aprenden, e incluso se redefinan, las estrategias que estas ponen en práctica.

De este modo, la elección de escuela, debe ser entendida al interior de dinámicas más amplias de reproducción cultural y social, en un ámbito en el que los límites entre agencia y estructura, autonomía y restricción, son difíciles de determinar. Asimismo, los motivos, valoraciones, expectativas de las familias, no pueden separarse del marco local y las particularidades de este.

En tal sentido, la focalización del análisis en la ciudad de Paraná, provincia de Entre Ríos, obedece a una cuestión de factibilidad pero también teórica. El subsistema privado de educación posee en el contexto provincial, características distintivas y singulares que ameritan considerar a la investigación como un estudio de caso, sin desmedro de que en algunas de las variables estudiadas pueda ser representativa de situaciones comunes al resto de las realidades provinciales, por ejemplo, en lo que refiere a la localización de escuelas privadas en zonas predominantemente urbanas (variable espacial), a la escasa regulación del sector por parte de los Estados provinciales (variable histórico-política) y a la falta de criterios objetivos para la adjudicación de subsidios a las escuelas privadas (variable histórico- político-económica). Con respecto al desarrollo del sistema educativo público de gestión privada en la provincia(2), los resultados de una investigación llevada adelante por Ossanna y López (2011) -el único antecedente específico sobre el mismo fenómeno y contexto en estudio- permiten corroborar que los gobiernos provinciales entrerrianos favorecieron el desarrollo de la educación privada durante el período 1976-1987. El cambio de tipo de gobierno no alteró sustancialmente esa actitud o coincidencia -tal como la denominan los autores- la cual corresponde más específicamente al nivel de la ideología (idea del valor de lo privado en relación con lo público, concepto de subsidiariedad del Estado, derechos de la familia sobre la educación de sus hijos) 
que al de la política partidaria. Las formas en las que el gobierno provincial favoreció al subsistema privado fueron diversas: adjudicación creciente de partidas presupuestarias para el pago de sueldos, subsidios y transferencia de bienes, ventajas legales a las instituciones privadas-autonomía creciente de los controles del Estado-, participación y cuasi control asegurado de los sectores privados en la administración pública de la educación privada, entre otros. El discurso de la subsidiariedad, la presión de las asociaciones patronales y la preponderante influencia de la Iglesia Católica, aparecen como respaldo teórico y empírico de la tendencia privatizante.

En cuanto a la escuela secundaria provincial, presenta un escenario con altos niveles de inequidad y uno de los desafíos más profundos en términos de política educativa provincial. Un documento de UNICEF (2011), sobre los indicadores educativos de la provincia de Entre Ríos, señala que entre los 12 y 14 años, la cobertura se ha incrementado entre 2001 y 2010 . En este último año, el 95,5\% de la población de este tramo asiste a la escuela. Sin embargo, en el tramo comprendido entre los 15 a 17 años, el 22, 2\% de la franja etaria no lo hace. El abandono y la retención de matrícula por repitencia reduce el flujo de alumnos grado a grado y solo el $33,4 \%$ de los alumnos que asisten con edad teórica a ler año llegan al último año de secundario sin repetir(3)

El documento de UNICEF indica que en el año 2010, aproximadamente uno de cada cuatro inscriptos en la primaria y secundaria asiste a un establecimiento de gestión privada. En el nivel inicial esta proporción se incrementa al $31 \%$ de los alumnos, y en el secundario orientado se sitúa en $27 \%$. Destaca el crecimiento sostenido en el nivel primario desde el 2002 en adelante, y el salto en la secundaria orientada entre 2004 y 2006 . Es probable, señala la misma fuente, que en el nivel secundario este incremento se deba al efecto conjunto entre un pasaje de alumnos del sector estatal al privado, junto con un incremento del abandono en las escuelas de gestión pública. En el caso del nivel inicial, este incremento del sector privado se debe al efecto conjunto de un pasaje de alumnos del sector estatal, junto con una mayor ampliación de cobertura en las edades tempranas ${ }^{(4)}$.

El análisis que a continuación se presenta indaga sobre los motivos de los padres que eligen el circuito privado de la educación para sus hijos. En tal
(3) En relación al año 200I, se evidencia también una mejora, que se reconoce principalmente en la disminución del porcentaje de población que no asiste y no accedió al título secundario, que desciende casi 6 puntos porcentuales entre el 200 y el 20Io. Sin embargo, algunos de los indicadores del nivel muestran cifras preocupantes, entre estos la sobreedad, que contribuye a perfilar las características de la progresión de los alumnos. En el inicio del secundario, se incrementa significativamente la cantidad de alumnos que asisten con sobreedad. En el $7^{\circ}$ grado asisten con edad teórica el 60\% de los alumnos, mientras que el $22 \%$ repitió una vez, y el I8\% repitió dos o más veces. $\mathrm{La}$ disminución de la matrícula entre $8^{\circ}$ y $9^{\circ}$ grado, y en los grados II y I2, es producto combinado del abandono y la retención de matrícula por repitencia, que reduce el flujo de alumnos grado a grado.

Como consecuencia de este desgranamiento, solamente 7.00o alumnos llegan al último grado del secundario sin repetir. Representan el $33,4 \%$ de los alumnos que asisten con edad teórica a Ier grado.

(4) Desde otra dimensión de análisis, relativa a los niveles de logro de los alumnos, el Informe, basado en los resultados de la prueba $\mathrm{ONE}$ 20Io, señala que las escuelas urbanas privadas muestran resultados significativamente más altos que las escuelas estatales, en todos los grados $\mathrm{y}$ áreas, con brechas que oscilan entre los 2I y 28 puntos porcentuales. 
(5) Desde el punto de vista de su territorio, el principal problema urbanístico que tiene la ciudad está constituido por los arroyos intra-urbanos, que conforman, en todos los casos, barreras de segregación. Siguiendo a Svampa (2008), definimos la segregación como la distancia -insalvable y permanente- entre los diferentes componentes de la sociedad, que muestra la consolidación de un modelo específico de socialización, basado en el contacto entre grupos homogéneos desde un punto de vista social y racial. Con excepción de los barrios que rodean el microcentro de la ciudad, el resto de los barrios presenta un empobrecimiento general, tanto en las condiciones de vida de sus habitantes como en los indicadores censales del barrio. Esta digresión interesa para comprender el mapa de las escuelas seleccionadas, porque asumimos que las escuelas difieren entre sí por su ubicación espacial y, al mismo tiempo, la ubicación espacial determina el nivel socioeconómico del alumnado. (6) El trabajo de campo fue llevado adelante durante los años 20I4-20I5 y constituyó el insumo de la tesis de doctorado titulada La escuela secundaria privada en la ciudad de Paraná (Entre Ríos), composición del campo y criterios de diferenciación, defendida y aprobada en el año 2016 . sentido, la escuela secundaria pública de gestión privada, en tanto dimensión de la desigualdad educativa, oferta una educación diferenciada que despierta motivos diferenciales en las familias, para proyectar el futuro de sus hijos. Hemos incluido en el análisis una referencia a los denominados migrantes, padres de hijos que comenzaron la escuela secundaria en una escuela pública pero que, por los motivos que exponen, cambiaron sus hijos a la oferta privada de educación.

\section{Las escuelas privadas en el contexto local: tradición y catolicismo, laicismo y mercado}

En la muestra de escuelas seleccionadas para el estudio, catolicismo, laicismo, tradición y mercado se encuentran en grados variables. El nivel socioeconómico de los estudiantes de las escuelas confesionales varía según la zona de localización y resulta relativamente homogéneo en cada una de ellas; pero el conjunto cubre un espectro que va desde la clase media hasta sectores de barrios muy carenciados ${ }^{(5)}$.

Dos variables intervinieron en el criterio de selección de escuelas: nivel socioeconómico del alumnado y carácter confesional o no confesional de las mismas. Se asume entonces, que los colegios difieren entre sí, porque atienden a poblaciones de distinto nivel socioeconómico (Alto-medio alto (Colegios D y A), Medio/ medio bajo (Colegio B), Medio bajo/ bajo (Colegio C). Durante la investigación ${ }^{(6)}$ fueron realizadas 27 entrevistas a padres y madres, apoderados, personal de los colegios, funcionarios y técnicos provinciales. Se incluyeron padres de establecimientos privados confesionales no incluidos en esta muestra y también padres que envían sus hijos a escuelas públicas de gestión estatal.El análisis realizado parte de considerar que los casos analizados, no son representativos de la variedad de opiniones posibles sobre el sistema educativo local o incluso sobre una misma escuela. Son representativos de puntos de vista de grupos específicos, ya que los padres no buscan, como los investigadores, contar y analizar este conjunto de opiniones para proponer interpretaciones sobre las representaciones de la escuela, sino para asegurarse de la pertinencia de su elección con respecto 
a familias y profesionales que comparten puntos de vista similares (Van Zanten, 2007, p. 257).

\section{La tradición familiar, la religión y la enseñanza en valores como los principales motivos de la elección}

La tradición familiar es el principal motivo de elección en los colegios privados católicos, dependientes de congregaciones religiosas que en la mayoría de los casos presentan un carisma particular (Ocampo, 2012) y que atienden a poblaciones de nivel socio-económico alto y medio alto. El Colegio A es un establecimiento con estas características. El alumnado es de nivel socioeconómico alto y medio alto y así lo ha sido desde su origen. El colegio funciona a dos cuadras del centro cívico de la ciudad de Paraná, en zona residencial. La escuela secundaria posee alrededor de 400 alumnos y recibe subsidios del Estado para el pago de los haberes de los docentes. Dirigido desde su creación en el año 1913 por los Hermanos de la orden, desde el año 1999 la dirección de la institución está a cargo de laicos. Este señalamiento no es menor, en tanto varios padres entrevistados, manifestaron un malestar muy grande sobre distintas dimensiones de la actual dirección, estableciendo notorias diferencias con la época en la cual los Hermanos estaban a cargo de la dirección del colegio. En tal sentido, resulta Ilamativo que en varios casos, se muestren claramente a favor de una dirección por el clero religioso antes que por laicos. Esta situación no es privativa del Colegio A. Consideramos que es un rasgo distintivo de la ciudad de Paraná, donde la Iglesia Católica tiene un fuerte peso, evidenciado entre otros indicadores, en la cantidad de católicos de acción, siguiendo la clasificación propuesta por Mallimaci (2015).

La tradición familiar es el motivo dominante de elección de escuela en los padres que envían sus hijos al Colegio $\mathrm{A}$. Todos estos padres poseen no solo capital económico, sino también cultural, social y simbólico. Son padres con nivel superior o universitario completo y se desempeñan en trabajos bien remunerados de la administración pública nacional, provincial o municipal y/o tienen emprendimientos privados. Una madre, ingeniera agrónoma, 
dueña junto a su familia de un importante emprendimiento agro-alimenticio de la ciudad de Paraná, señala:

En el momento que elegimos, mi esposo había ido a escuela privada, yo había ido a escuela privada, y, bueno, por eso elegimos por la educación privada. (...) Y el Colegio $\mathrm{A}$ específicamente (...) mi esposo era ex alumno, muy contento con eso, y bueno, por eso (Madre 2, Colegio A)

En general, la mayor parte de los padres son ex-alumnos del colegio. Para ellos, el Colegio A garantiza una educación en valores, que adquiere distintas connotaciones al momento de ser definida. Para algunos, alcanza una dimensión moral, para otros, se encuentra más cercana a una dimensión social:

Uno prioriza (...) la educación en los valores, en el compartir, en el respeto, en el ver en el otro lo que necesita. Y creo que en eso la escuela hace mucho hincapié. (...) Porque incluso los docentes eran muchos de los mismos que había tenido mi esposo en ese momento (Madre 2, Colegio A)

El colegio elegido es la prolongación de la casa. La importancia de la educación de la escuela es relativizada en función de la educación de la casa, lo cual reproduce a nivel opinión de los padres el principio de subsidiariedad estatal, según el cual el Estado debe cumplir un rol secundario o complementario frente a otros agentes del ámbito privado, como las familias o las instituciones religiosas.

\section{Variaciones en la formación: formación integral y formación religiosa}

En cuanto al sentido de la formación religiosa, si para algunos padres pasa por una cuestión de comodidad en el sentido de "ahí tenemos todo", se trata para otros de una formación más integral, sustentada en valores cristianos, en un estilo de trabajo particular signado por la convivencia. Lo cierto es que la cuestión amerita complejizar el sentido de tal afirmación, habida cuenta que la formación en valores religiosos surge como un motivo importante, 
tanto en padres que envían sus hijos a las escuelas de la muestra como a otros colegios privados católicos.

Lo advertido en primer lugar, acerca de una cuestión cómoda y económica aparece señalado una madre:

Bueno, ¿ves?, eso tampoco no te dije. A los chicos les enseñan catequesis ahí, les hacen tomar la confirmación, la comunión. Yo no tengo que mandarlo extra a Lucas a ningún lado. (Madre 6, Colegio C)

Sin embargo, varios padres del Colegio A, refieren a la formación religiosa como una formación integral, que articule los valores cristianos con el compromiso social. Se vincula por ejemplo, con la Pastoral, un tipo de acción que suma al asistencialismo, las tareas de monitoreo, planificación y ejecución de planes sociales provenientes del Estado (Mallimaci, 2015). Se trata de padres con preocupaciones sociales, que enfatizan la fuerte dosis de compromiso social de esta actividad:

Bueno, a la tarde [contesta acerca de si la escuela ofrece actividades extra-curriculares] solamente educación física, ahora contraturno, y después una oferta más religiosa, cuasi social religiosa que es la Pastoral, que ellos hacen convivencia, actividades lúdicas y terminan sexto año misionando en el norte, apadrinan a algunas escuelas del norte y después, bueno, ellos van y dan algún tipo de servicio. [Le pregunto si esa dimensión es importante para su hija] Sí, sí, sí. Ella participó el año pasado y quedó chocha porque ella, bueno, es muy crítica a su vez de lo religioso (Madre 1, Colegio A)

Estos padres se piensan a sí mismos y a sus hijos con conciencia social y valoran que estos se formen en un ambiente heterogéneo, tanto desde el punto de vista social como cultural:

Saber que era una escuela privada pero muy heterogénea en su conformación, en los grupos; sabíamos que había de todo. Estaban gente con mucha plata, pero también sabíamos que había gente de humilde para abajo, y nos gustaba esa mezcla (Madre 1, Colegio A) 
Resta mencionar aún, un tercer grupo de padres que, siguiendo a Mallimaci (2015) denominamos católicos de acción. Estos, señala el autor, suelen sentirse decepcionados, porque se dan contenidos, pero no se insiste en la vivencia de la fe, tal como lo indican los siguientes testimonios:

(...) nosotros somos católicos practicantes. Y entonces nuestro primer interés fue ese, mandarlos a una escuela religiosa. Nos decepcionamos mucho en ese sentido, porque no era lo que esperábamos en cuanto a la religión, digamos. Pero en cuanto a la parte educativa yo estoy re conforme (Madre 4, Colegio A)

\section{La elección basada en la contención social y la disciplina}

En el caso del Colegio $C$, una institución pública de gestión privada católica, dependiente del Arzobispado de Paraná, que atiende a poblaciones de nivel socioeconómico medio bajo, bajo y marginal, la elección comprende tres dimensiones: 1) buscar una alternativa a la escuela pública, 2) que en la escuela haya disciplina y 3) que sea un ámbito de contención social. A pesar de tener una escuela pública a dos cuadras de su casa, esta madre -empleada doméstica, esposo policía- envía su hija a la escuela C, a treinta cuadras de su casa. Señala de la experiencia en la escuela pública de gestión estatal, a la cual concurrieron -durante los primeros años de escolarizaciónsu hijo mayor y también su hija:

Y, la escuela privada, en mi caso, digamos, tengo una [escuela pública] cerca de mi casa que, por ejemplo, yo llevaba los chicos a la escuela, nunca tenían clase, es muy 11) lento el [aprendizaje] (Madre 5, Colegio C)

De todos modos, gran parte de su familia,-que vive en la zona de la escuela C-, asistió a la misma, de modo que enviar su hija a esta, fue un desenlace natural luego de la experiencia de sus primeros hijos. Se muestra conforme con su elección y manifiesta, además, la importancia que tiene, para ella y su marido, que sus hijos reciban educación religiosa dentro del mismo 
establecimiento educativo al que concurren, porque de ese modo no deben mandar a los niños por separado a catequesis. También destacó el bajo costo de la cuota.

Con referencia a la disciplina, una madre señala:

Y, la educación; que las maestras no faltan; la disciplina; en la enseñanza que les hacen; que no hay peleas; el vocabulario (...) Catequesis. Mucha retención, los retienen mucho a los chicos. Las docentes te ayudan mucho, colaboran con los chicos. Y después hay mucha disciplina, mucha disciplina (Madre 6, Colegio C)

Esta última opinión pone en evidencia que para poblaciones de nivel económico medio-bajo, bajo y marginal, la escuela privada parroquial, es una opción, en el sentido postulado por Gamallo (2007), al afirmar que la 'fuga` hacia la escuela privada, producida sobre todo a finales del año 2002, incluye a alumnos provenientes de los quintiles de ingresos más bajos.

Si continuamos con el análisis, identificamos como dimensiones del mismo concepto de disciplina, la referencia de los padres a la organización interna de la escuela: el cumplimiento en los horarios de entrada y salida y durante la jornada escolar, la dimensión burocrática-administrativa y en menor medida, el uso de un uniforme escolar. Una consecuencia de lo anterior, es la seguridad con la cual los padres envían sus hijos a la escuela, traducida en el hecho de no poder retirarlos en cualquier momento y por cualquier persona:

La diferencia notable es la organización interna y la rigidez frente a ciertas normas que son normas comunes que uno aplica cuando va a una escuela privada o que uno aplica cuando dice, bueno, a ver, el uniforme. En las escuelas públicas tenés uniforme, pero no te obligan; tenés horario de entrada, pero son flexibles; tenés la puerta abierta; formación a la bandera se hace una especie de formación. (Padre 4, otra escuela privada católica, se desempeña como docente en una escuela técnica de la ciudad)

En cuanto a la contención social presenta distintos matices. Uno está dado por el acompañamiento psicopedagógico y psicológico de la institución: 
Hay de todo un poco, hay tanto padres separados, padres que son adictos a la droga, eh... hay chicos que, ¿cómo se llama?, han muerto papá y mamá y la abuela se hace cargo del hijo. Hay chicos complicados también; pero hay un grupo de psicólogos, psicopedagogas que ayudan mucho a los chicos (Madre 6, Colegio $\mathrm{C}$ )

Sin embargo, más allá de este acompañamiento, existe un seguimiento más bien tutorial, basado en la escucha a los alumnos. En el caso de la Escuela $\mathrm{C}, \mathrm{y}$ tal como fue relatado por su secretario, en la misma entrevista inicial a los futuros docentes, se les explicita que el objetivo de la escuela es ayudar y acompañar al alumno.

\section{Queríamos una escuela laica, chica y sin paros}

La escuela B es un establecimiento privado, creado y administrado por personas físicas, con fines de lucro, que atiende a poblaciones de nivel socio-económico medio y medio bajo -empleados públicos y empleados de comercio- aunque también algunos funcionarios y empleados del gobierno.. En el año 1996, se puso en funcionamiento la sala de cinco años y a partir de este año, se fueron abriendo los cursos. La primera promoción de egresados de sexto año -modalidad bachiller con orientación en economía y gestión de la organización- fue en el año 2008.

Los motivos de elección de escuela expuestos por estos padres, se vinculan de forma negativa con las carencias de la escuela pública de gestión estatal, antes que por ventajas explícitas de la institución de referencia. Al mismo tiempo, ponen de relieve la importancia de canales informales de comunicación en el medio local, donde la información sobre la escuela se adquiere más por vínculos en el ambiente laboral que por un contacto directo con la institución:

Nosotros buscábamos, inicialmente pensábamos en la escuela pública. El tema era los paros y la falta [de] los días perdidos de estudios. Y él [se refiere a su marido], no quería una escuela religiosa, quería una laica; entonces, cuando le tocó a Franco empezar la escuela él trabajaba en el SAIE, entonces se enteró de esta escuela que estaba formándose, 
porque era el segundo año que trabajaba, y tenía buena referencia. Es más que nada por ese tema, [aclara] de los paros y las pérdidas, los días perdidos que no se recupera en los chicos (...) Así que es él [se refiere a su marido] el que quería una escuela chica, que era nueva, y que no había paros (Madre 8 , Colegio B)

La elección por ser una escuela chica está vinculada al interés de los padres porque sus hijos reciban una atención personalizada, similar al caso anterior. Al igual que en otras escuelas privadas no confesionales, que surgieron en la década del noventa -en la cual la reconfiguración de la educación otorgó legitimidad a la educación como objeto de beneficio económico, de manera contratable y vendible-, en esta escuela es visible una disminución en la calidad de la oferta académica. No solo faltan los docentes -indican los entrevistados- sino que ha disminuido también la calidad educativa de ciertos espacios curriculares que supieron ser de excelencia en la década del noventa:

Él tuvo una buena base de matemática [se refiere a la educación que recibió su primer hijo, que hizo la escolaridad entre los años 1996- 2009], que le sirvió un montón en la facultad; una buena base de inglés, porque tienen un montón de trabajos que hacer en inglés y lo hace todo en inglés y no fue más que a la escuela, no fue a maestra particular ni a ningún lado. $Y$ cuando él iba yo no me acuerdo que haya habido tampoco mucha falta de docentes; ahora con Sofía sí faltan más los docentes en la secundaria. Faltan docentes y tienen horas libres. Con Franco no era así, no faltaban así los docentes, era raro. (Madre 8 , Colegio $\mathrm{B}$ )

En cuanto a la matrícula de este establecimiento, si bien nunca numerosa, ha disminuido en forma notable en los últimos años, egresando en el año 2015 solo siete alumnos.

\section{El fortalecimiento de redes y lazos sociales como motivos predominantes}

El Colegio D, es una escuela pública de gestión privada, creada y administrada por personas físicas, con fines de lucro, que atiende a poblaciones 
de nivel socio-económico medio alto y alto. La mayoría de los padres son profesionales, con nivel universitario completo, con ejercicio en la actividad privada y con emprendimientos también privados. Al igual que el colegio $B$, su creación data de la década del noventa, año 1993.

El alumnado del colegio $D$ es la manifestación más acabada de la afirmación de Cervini $(2002,2003)$ al señalar que la incidencia de la herencia cultural familiar del estudiante individual, opera a través del agrupamiento en instituciones escolares. Los culturalmente iguales tienden a estar institucionalmente juntos, subraya el autor y en este caso, el colegio presenta la mayor homogeneidad social de todas las instituciones analizadas.

Un motivo es dominante en la elección de escuela: fortalecimiento de capital cultural y de capital social. Los padres persiguen no solo un interés formativo sino también social, evidenciado en la utilidad que las redes sociales construidas en el tránsito por la escuela, sirvan en el futuro, para su hijo o hija. Tal como sostenía una docente de arte, que se desempeña en tres escuelas privadas de la ciudad, madre a su vez de una hija en la escuela $D$, los alumnos se mueven en grupos que casi parecen tribus, y esto sucede todo el tiempo, trascendiendo el tiempo escolar, próximos en el espacio social:

Estos grupos [se refería a bandas de punks mientras dictaba una clase] se manejan con determinados códigos, al igual que ustedes se manejan con determinados códigos, ustedes son una tribu también; más allá de esa crítica que uno hace, que si usan, que si no usan, que si tienen ritos que a nosotros nos parecen extraños, este... ustedes hacen esto, esto; porque son eso, son como tribus, todos van al mismo club, todos se compran la ropa en los mismos lugares (Docente y madre 14, Colegio D)

Aún en el caso de alumnos que se han cambiado de una escuela privada a otra -en este caso, la madre da clases en el colegio A y notaba que la calidad de la educación era en esta, mucho más alta que en la escuela a la cual asistía su hija- la relación con los nuevos compañeros fluye de manera natural:

Entonces, a la semana empieza ya allá en [el Colegio A], los amigos que son del club, del Rowing, eh... la esperan en la puerta un rato antes, la hacen sentar al lado, o sea, la integran inmediatamente. $Y$ bueno, ella termina tercero, cuarto y quinto en el colegio [A]. 
$O$ sea, no hizo toda su trayectoria, sino que la hizo inicialmente en el Cristo y después pasó a [Colegio A] (Docente y madre 13, Colegio A)

\section{Los migrantes: la escuela privada como segunda opción}

Aún en padres de nivel socioeconómico medio y capital cultural, la escuela estatal es referida como la primera opción en la cual pensaron al momento de elegir la escuela de sus hijos. Los testimonios evidencian que en gran parte, la situación de deterioro de las escuelas estatales a raíz de paros y días sin clases, no es nueva, y se remonta a la década del noventa, cuando iniciaron su escolaridad los hijos mayores de algunos padres entrevistados, que hoy tienen en promedio 25 años.

En todos estos padres, opera una auto-justificación de la opción elegida, en parte porque muchos de ellos fueron a escuelas estatales, en parte por una valoración del Estado como principal responsable de la educación.

Nosotros buscábamos, inicialmente pensábamos en la escuela pública. El tema era los paros y la falta [de] los días perdidos de estudios. Es más que nada por ese tema, [aclara] de los paros y las pérdidas, los días perdidos que no se recupera en los chicos (Madre 8, Colegio B)

A la falta de días de clase por distintos motivos: paros y asambleas docentes, fumigación, factores climáticos, este padre suma otro elemento más, vinculado a la dimensión organizacional de la institución y a la seguridad que estos padres migrantes encuentran en la escuela privada:

Lo otro bueno que tiene esa escuela es que nadie sale sin permiso. Vos entrás a la escuela y ya sos parte de la escuela, si querés salir te anotan, te registran, o te vienen a buscar (Padre 4, otro colegio privado católico)

La migración puede darse también por la falta de respuestas frente a dificultades particulares tales como síndromes, discapacidades u otras. En el caso de este padre, encontró en la escuela privada el ámbito propicio para 
la inserción de su hijo diagnosticado con Asperger. Recuerda que cuando su hijo empezó el jardín de infantes lo querían hacer repetir jardín, y fue el momento en que iniciaron distintos tratamientos psicopedagógicos y cambiaron al niño a una escuela privada:

La contención que tuvo en [la escuela privada católica a la cual lo cambiaron], a nivel psicopedagogo, a nivel maestras, con respecto a lo que venía en [la escuela estatal] la cuestión de que siempre le falta algo, veo que hay predisposición de los profesores, vos te podés acercar a la escuela a charlar y no es una escuela muy cerrada, podés tener el acceso a cada profesor, siempre tenés que tener acceso mediante nota, o sea, la formalidad ellos, la formalidad y la burocracia la tienen muy presente (Padre 4, otro colegio privado católico)

La contención toma la forma en estos casos, de atención personalizada. El acompañamiento y el seguimiento son aspectos muy valorados por los padres. Se infiere también que valoran la motivación y compromiso de los docentes con el aprendizaje de los alumnos al mismo tiempo que se observa una sensación de control directo a los docentes, realizada tanto por los padres como por la dirección de la misma escuela.

Con excepción de los padres que han elegido la escuela para sus hijos por tradición familiar, la mayor parte de los padres entrevistados asistieron a establecimientos públicos de gestión estatal en el ámbito local. Ello se explica en parte, porque hasta la década del noventa no hubo en la ciudad de Paraná demasiadas escuelas privadas secundarias -en su mayoría las privadas eran las pertenecientes a las congregaciones religiosas- pero también porque la educación privada fue una opción solo para las familias que contaban con recursos suficientes para garantizar no solo el pago de una cuota -aunque fuera de bajo costo-, sino también otros gastos: uniforme, traslado, útiles escolares.

Un padre señala que cuando él inició la escuela secundaria, lo cercano y lo barato fue la única opción. Cuando los tiempos mejoraban y si la economía familiar lo permitía, algún hermano asistía a escuela privada: 
Mi hermana, por ejemplo, ya hizo... yo con mi hermana me llevo siete años de diferencia, hizo privada primaria y secundaria (...) ella se mantuvo, digamos, en lo privado por una cuestión de que ya había un poco más de recursos (Padre 4, otro colegio privado católico)

El testimonio prueba que el crecimiento de la oferta privada sucede a la par del mejoramiento en las condiciones económicas del país y de la región. Al mismo tiempo, pone en evidencia uno de los motivos del crecimiento sostenido de la educación privada católica de bajo costo, habida cuenta de las características del medio local, una ciudad en la cual la mayor parte del empleo es proveído por el Estado nacional, provincial o municipal.

\section{A modo de conclusión}

La evidencia empírica ha demostrado que los padres no solo elaboran verdaderas estrategias de elección de escuelas, sino que además, ponen en marcha iniciativas propias.

En alguna dimensión de análisis, el caso local presenta semejanzas con la descripción que la bibliografía internacional hace de la elección de padres de clase media. Por ejemplo, Olmedo Reynoso y Santa Cruz Grau (2008), señalan que las familias de clase media primero eligen el tipo de producto particular que quieren y después se deciden por la escuela concreta que les ofrece el producto. Este es el caso de muchos padres entrevistados, quienes manifestaron el motivo dominante por el cual seleccionaron la escuela, que dio origen luego, a la decisión final sobre la misma.

En el caso analizado, los motivos dominantes son la tradición familiar y la enseñanza en valores (Colegio A), ser una alternativa a la escuela pública, la disciplina y la contención social -aunque también la enseñanza en valores religiosos- (Escuela $\mathrm{C}$ ), laicidad y el carácter chico de la institución (Escuela B) y el fortalecimiento de capital cultural, social y simbólico en la escuela de más alta homogeneidad social de la muestra (Colegio D). Los padres entrevistados manifiestan que existe en las escuelas privadas organización interna, disciplina y cumplimiento de normas. Perciben que los docentes de las instituciones privadas están controlados por los mismos padres y por la 
dirección, que en cada escuela asume un estilo diferente. En este marco, cobra sentido la tarea de acompañamiento que -hemos supuesto- permite activar el capital cultural de los alumnos en las escuelas privadas que atienden a poblaciones con niveles socioeconómicamente bajos y marginales. Aunque no en todos los casos, surge de las entrevistas una auto-justificación de los padres de clase media que envían sus hijos a escuelas privadas, aludiendo al no cumplimiento de la función básica de la escuela pública de gestión estatal, esto es, el no dictado de clases por distintas causas. La mayor parte de los padres entrevistados asistieron a escuelas públicas de gestión estatal, lo cual pone en evidencia que el mercado de la educación local, es un fenómeno relativamente reciente, que puede ubicarse en la década del noventa.

Sin embargo, fue en el período posterior a la crisis acaecida en diciembre del año 2001 cuando comenzó el éxodo de los sectores medios y medios bajos a la escuela privada. Durante esta década, elementos propios del mercado se recuperaron y se introdujeron en el ámbito educativo: la libre elección de los padres a elegir la oferta educativa más adecuada para sus hijos, la autonomía de las escuelas y la competencia entre ellas, evidenciada en la necesidad de captar clientes en un reducido mercado local.

Todo parece indicar, que a pesar de la pérdida gradual del poder adquisitivo -motivada entre otros factores por la elevada inflación de los últimos añosde la clase media local, compuesta en su mayor parte por asalariados del sector público y privado del sector servicios, estos sectores no han retornado a la escuela pública estatal.

Sin embargo, otras causas pueden invocarse también. Hoy la clase media local no envía sus hijos a la escuela pública estatal y, del mismo modo que se ha diversificado y no constituye un estrato social homogéneo, se ha diversificado también la oferta escolar. Parafraseando a Wortman (2009), la clase media sigue creyendo que la educación es prioritaria para el ascenso social, pero percibe que esta debe ser privada. La educación pública es considerada un espacio social inseguro, más vinculado a la contención social de alumnos difíciles, que a la formación del capital cultural y social. En el contexto local, la escuela secundaria presenta evidencias de un profundo proceso de fragmentación, en el cual los alumnos de los distintos 
colegios privados entre sí y con colegios de la oferta estatal, pertenecen a mundos culturales que difieren entre sí en virtud de los valores, las expectativas y los modos de vida que los organizan (Tiramonti y Ziegler, 2008, p. 29). En tal sentido, la heterogeneidad social -que supo tener la escuela pública en la ciudad, hasta fines de la década del ochenta -según testimonios de los padres entrevistados- va en vías de extinción.

\section{Referencias bibliográficas}

Bourdieu P. (2011). Las estrategias de la reproducción social. Buenos Aires: Siglo Veintiuno Editores.

Cervini, R. (septiembre-diciembre 2002). Desigualdades en el logro académico y reproducción cultural en Argentina. Un modelo de tres niveles. Revista Mexicana de Investigación Educativa, 7(16), 445-500. Recuperado de: http://www.quadernsdigitals.net/datos/ hemeroteca/r_54/nr_603/a_8218/8218.pdf

Cervini, R. (febrero, 2003). Diferencias de resultados cognitivos y no-cognitivos entre estudiantes de escuelas públicas y privadas en la educación secundaria de Argentina: Un análisis multinivel. Education Policy Analysis Archives, 11(5). Recuperado de: http://epaa. asu.edu/epaa/v11n6/

Gamallo, G. (2011). Mercantilización del bienestar. Hogares pobres y educación privada. Revista de Instituciones, familias y mercados, 55, 189-233. Recuperado de: https://www.eseade.edu.ar/wp-content/ uploads/2016/08/riim55_9_gamallo.pdf

Gutierrez A. (2005). Las prácticas sociales, una introducción a Pierre Bourdieu. Córdoba: Ferreyra Editor.

Gutierrez A. (2011). Clases, espacio social y estrategias. Una introducción al análisis de la reproducción social. En P. Bourdieu (2011). Las estrategias de la reproducción social (pp. 9-22). Buenos Aires: Siglo XXI Editores.
Mallimaci, F. (2015) El mito de la argentina laica. Catolicismo, política y estado. Ciudad Autónoma de Buenos Aires: Capital intelectual.

Ocampo, M. (2012). Modelos escolares católicos y cultura escolar. Página Educativa, 5(1), 55-78. Recuperado de: htttp://www.scielo.edu.uy/pdf/pe/v5n1/ v5n1a04.pdf

Olmedo Reynoso A. Santa Cruz Grau E. (2008) Las familias de clase media y la elección de centro: el orden instrumental como condición necesaria pero no suficiente. Profesorado. Revista de currículum y formación del profesorado, 12(2) 1-30. Recuperado de http://www. ugr.es/local/recfpro/rev122ART7.pdf

Ossanna E. Y López M. P. (2011). Educación, sociedad y política: la escuela privada en Entre Ríos, 1976 1987. Entre Ríos: Facultad de Ciencias de la Educación, Universidad Nacional de Entre Ríos.

Rivas, A. (2004). Gobernar la educación: estudio comparado sobre el poder y la educación en las provincias argentinas. Buenos Aires: Granica.

Svampa M. (2008). Los que ganaron: la vida en los countries y en los barrios privados. $2^{a}$ edición. Buenos Aires: Biblos.

Tiramonti G. (comp.). (2007). La trama de la desigualdad educativa. Mutaciones recientes en la escuela media. Buenos Aires: Manantial. 
Tiramonti, G., Ziegler, S. (2008). La educación de las elites: aspiraciones, estrategias y oportunidades. Buenos Aires: Paidós.

UNICEF. (2011). La educación en cifras. Provincia de Entre Ríos. Indicadores seleccionados para la caracterización del sistema educativo.

Van Zanten A. (2007). Reflexividad y elección de la escuela por los padres de la clase media en Francia. Revista de Antropología Social 245(16), 245-278.

Veleda C. (2007). Entre querer y poder. Las clases medias y la elección de la escuela en el conurbano bonaerense, en M. Narodowski y M. Gomez Schettinni: Escuelas y Familias. Buenos Aires: Prometeo.

Wortman A. (agosto/noviembre, 2009). La clase media argentina ya no existe. Muros de la cultura, nuevas tecnologías y desigualdad social. Revista Umbrales de América del Sur, 9. Buenos Aires, Argentina: CEPES. 